\title{
Identificación molecular con base en el gen hsp70 de aislamientos clínicos de Leishmania spp. en Colombia
}

\author{
Ana M. Montalvo', Jorge Fraga1', Ivón Montano', Lianet Monzote¹, \\ Gert Van der Auwera², Marcel Marín ${ }^{3}$, Carlos Muskus ${ }^{3}$ \\ 1 Departamento de Parasitología, Instituto de Medicina Tropical Pedro Kourí, La Habana, Cuba \\ 2 Department of Biomedical Sciences, Institute of Tropical Medicine, Antwerp, Belgium \\ 3 Programa de Estudio y Control de Enfermedades Tropicales, PECET, Facultad de Medicina, Universidad de \\ Antioquia, Medellín, Colombia
}

Introducción. La leishmaniasis es una enfermedad de gran prevalencia en Colombia, donde al menos seis especies diferentes la originan en el ser humano, con un amplio rango de características clínicas. La tipificación de la especie es importante, no solo desde el punto de vista epidemiológico, sino para el diagnóstico, dado que el tratamiento puede variar dependiendo de la especie identificada. En la identificación se han utilizado varias alternativas metodológicas con diferentes niveles de poder discriminatorio.

Objetivo. Identificar especies de Leishmania mediante la amplificación molecular de un fragmento del gen $h s p 70$.

Materiales y métodos. Se amplificó un fragmento del gen hsp70 mediante reacción en cadena de la polimerasa (PCR-hsp70) y se hizo el análisis de los polimorfismos de la longitud (Restriction Fragment Length Polymorphism, RFLP) de los fragmentos de restricción de 81 aislamientos clínicos de Leishmania spp. de pacientes con leishmaniasis cutánea y mucocutánea para la identificación de las especies presentes.

Resultados. Se obtuvo un solo amplicón para todas las muestras analizadas. La restricción enzimática de los 81 productos permitió la identificación de 70 con dos patrones de bandas que correspondían a dos alelos de Leishmania braziliensis (62 y ocho aislamientos, respectivamente), nueve aislamientos compatibles con L. panamensis y dos con L. guyanensis. El origen geográfico de los aislamientos coincidió con el de reportes previos sobre la distribución de dichas especies en Colombia.

Conclusiones. La técnica de la PCR-hsp70 y el análisis de RFLP fueron útiles para identificar las especies de Leishmania aisladas de muestras clínicas de Colombia y pueden aplicarse también en el estudio de cepas de vectores y reservorios de importancia epidemiológica.

Palabras clave: Leishmania, diagnóstico, reacción en cadena de la polimerasa, polimorfismo de longitud del fragmento de restricción.

doi: http://dx.doi.org/10.7705/biomedica.v36i2.2688

Molecular identification of Leishmania spp. in clinical isolates from Colombia based on hsp70 gene

Introduction: Leishmaniasis is highly prevalent in Colombia, where at least six different species can cause disease of varying clinical presentations in humans. The identification of the infecting species is quite important for prognosis, therapeutics and epidemiology. Different techniques with variable discriminatory power have been used for the identification.

Objective: To carry out the molecular identification of Leishmania species through the amplification of a fragment of the $h s p 70$ gene.

Materials and methods: Molecular amplification of the hsp70 gene fragment (PCR-hsp70) followed by restriction fragment length polymorphism analysis (RFLP) was done for identification purposes using DNA from 81 clinical isolates of Leishmania.

\footnotetext{
Contribución de los autores:

Ana M. Montalvo: concepción, diseño, ejecución, análisis de los resultados y escritura del documento Jorge Fraga: ejecución y análisis de los resultados Ivón Montano y Lianet Monzote: ejecución e interpretación de los resultados

Gert Van der Auwera: análisis e interpretación de los resultados

Marcel Marín: recolección de datos y muestras; ejecución

Carlos Muskus: concepción, análisis e interpretación de los resultados

Todos los autores participaron en la revisión del manuscrito.
} 
Results: A single amplicon was obtained for all samples analyzed. The enzymatic restrictions of the 81 PCR products identified 70 with a banding pattern corresponding to L. braziliensis with two different patterns (62 and eight isolates, respectively), nine isolates compatible with $L$. panamensis and two with $L$. guyanensis. The geographical origin of the isolates is consistent with previous reports about the distribution of the corresponding species in Colombia.

Conclusions: The PCR-hsp70/RFLP technique used is a valid tool for the identification of Leishmania species isolated from clinical samples of patients in Colombia, which may also be applicable to the study of strains obtained from vectors and reservoirs with epidemiological significance.

Key words: Leishmania, diagnosis, polymerase chain reaction, polymorphism, restriction fragment length. doi: http://dx.doi.org/10.7705/biomedica.v36i2.2688

La infección del hombre por parásitos del género Leishmania, de amplia distribución mundial, puede producir leishmaniasis, un complejo de enfermedades presentes en todas las áreas geográficas, principalmente en países tropicales y subtropicales, donde 350 millones de personas se encuentran en riesgo de contraerla. Se estima que cada año hay 1,5 a 2 millones de nuevos casos, de los cuales 500.000 corresponden a la forma visceral $y$, el resto, a casos de leishmaniasis cutánea y, en un porcentaje menor, a leishmaniasis mucocutánea, cifras que, aunque se aceptan actualmente, continúan en revisión (1).

Teniendo en cuenta la variedad de especies que pueden ser patógenas para el hombre y las diferentes condiciones geográficas en las que se dan, la leishmaniasis se puede considerar como un grupo polimorfo de enfermedades complejas, con características y opciones de tratamiento y pronóstico diferentes (2).

La variedad en la presentación clínica depende, por lo general, de varios factores, entre los cuales la propensión genética del huésped, el contexto inmunológico en que se produce la infección $(3,4)$ y la especie de Leishmania $(5)$, son los más importantes. Es por ello que la determinación de la especie infecciosa ha pasado a ser un requisito esencial para el diagnóstico y el tratamiento (6), así como para la comprensión de aspectos epidemiológicos fundamentales como la identificación de especies simpátricas o importadas, las especies asociadas con el fracaso del tratamiento, la incriminación y la asociación de

\section{Correspondencia:}

Carlos Muskus, Programa de Estudio y Control de Enfermedades Tropicales, PECET, Facultad de Medicina, Universidad de Antioquia, Carrera $53 \mathrm{~N}^{\circ}$ 61-30, laboratorio 632, Sede de Investigación Universitaria, Medellín, Colombia

Teléfono: (574) 2703442

carmusk@yahoo.com

Recibido: 06/02/15; aceptado: 09/09/15 posibles vectores y parásitos, o la determinación de la presencia de especies híbridas, entre otros (7).

En la identificación de especies se utilizan los métodos moleculares debido a su gran sensibilidad para detectar el parásito infeccioso en muestras de humanos, animales vertebrados e insectos vectores. Las amplificaciones basadas en la reacción en cadena de la polimerasa (PCR) han utilizado diversos blancos moleculares, como los espaciadores internos de la transcripción del ARN ribosómico (ITS-rRNA) (8), el ADN del cinetoplasto (kDNA) $(9,10)$ y el gen que codifica la proteína de choque térmico de $70 \mathrm{kDa}$ (hsp70) (11), cuya utilidad se ha comprobado ampliamente en años recientes.

Esta identificación puede hacerse de manera directa con una PCR específica para la especie (12), o mediante procedimientos posteriores a la amplificación molecular, como el análisis del tamaño de los fragmentos de restricción (Restriction Fragment Length Polymorphism, RFLP) (13-15). En este contexto, la variante PCR-hsp70/RFLP basada en el gen hsp70, propuesta por García, et al. (11), y modificada posteriormente, detecta 14 especies de importancia médica usando un solo par de cebadores y, a partir de un único producto de amplificación, logra identificar individualmente la mayoría de dichas especies mediante una o dos restricciones enzimáticas (15). Esta variante se ha aplicado tanto al análisis de cepas como al de muestras clínicas humanas (16).

En Colombia se han reportado, al menos, seis especies de Leishmania distribuidas en el territorio: L. panamensis, L. braziliensis, L. guyanensis, $L$. mexicana y $L$. amazonensis, asociadas con las formas cutánea y mucocutánea de la enfermedad, y L. infantum (L. chagasi), asociada principalmente a la forma visceral (17). Asimismo, se han aplicado distintos protocolos moleculares para la identificación de estos parásitos en cepas de referencia y aislamientos de casos clínicos $(18,19)$. 
En este contexto, el objetivo de este trabajo fue la identificación molecular de especies de Leishmania provenientes de distintas zonas de Colombia mediante la aplicación de la técnica de PCRhsp70/RFLP a un grupo de aislamientos clínicos no caracterizados, lo cual podría ser de interés para el manejo de esta enfermedad y el conocimiento de la biología de su agente etiológico.

\section{Materiales y métodos}

\section{Aislamientos clínicos}

Se utilizaron parásitos cultivados obtenidos de 81 muestras de biopsias y raspados de pacientes con leishmaniasis cutánea, provenientes de distintas áreas geográficas del país y atendidos en el Programa de Estudio y Control de Enfermedades Tropicales (PECET) de la Universidad de Antioquia en Medellín, Colombia. Para mantener los parásitos in vitro se incubaron a $26{ }^{\circ} \mathrm{C}$ en medio Schneider (SIGMA, St. Louis, MO, EUA) con suplemento de $10 \%$ de suero fetal bovino, inactivado por calor a $56{ }^{\circ} \mathrm{C}$ con una mezcla de estreptomicina $(250 \mu \mathrm{g} /$ $\mathrm{ml}$ ) y ampicilina (100 UI).

\section{Obtención del ADN a partir de los aislamientos clínicos}

Para la extracción del ADN se utilizó el método descrito por Sambrook, et al. (20), a partir de los parásitos obtenidos de los aislamientos.

\section{Cebadores para la amplificación del gen hsp70}

Se utilizaron los cebadores propuestos por García, et al. (11), los cuales amplifican un fragmento polimórfico de $1.422 \mathrm{pb}$ del gen hsp70 de Leishmania spp. y permite la tipificación de numerosas especies de Leishmania mediante digestión enzimática (15). Los cebadores se sintetizaron con SIGMA Genosys (Bornem, Bélgica), cuyas secuencias (5'-3') son la positiva para hsp70: 5' GACGGTGCCTGCCTACTTCAA 3' y la negativa para hsp70: 5' CCGCCCATGCTCTGGTACATC 3'.

\section{Protocolo de la PCR-hsp70}

La mezcla de reacción $(50 \mu l)$ contenía $200 \mu \mathrm{M}$ de cada trifosfato de desoxirribonucleótido (dNTP) (Promega, EUA); 1X de la solución tampón (Tris$\mathrm{HCl} 670 \mathrm{mmol} / \mathrm{l} \mathrm{pH=8,8;}\left(\mathrm{NH}_{4}\right)_{2} \mathrm{SO}_{4} 160 \mathrm{mmol} / \mathrm{l}$; Tween 20 0,1\%); $2,5 \mathrm{mmol} / \mathrm{L}$ de $\mathrm{MgCl}_{2} ; 1 \mathrm{U}$ de la enzima SilverStar TaqDNA polimerasa (Eurogentec, Seraing, Bélgica) y $0,4 \mu \mathrm{mol} / \mathrm{L}$ de cada cebador, además de dimetilsulfóxido al 0,25\% (DMSO) y $100 \mathrm{fg}$ del ADN obtenido de los aislamientos. Se incluyó en todos los experimentos agua destilada como control negativo y, ADN de L. braziliensis, cepa MHOM/BO/--/CUM180, como control positivo. Las reacciones de amplificación se hicieron en un termociclador PTC-0150 Mini-Cycler (MJ Research, Waltham, MA, EUA).

El protocolo consistió en la desnaturalización inicial (a $94^{\circ} \mathrm{C}$, durante 5 minutos) seguida de 33 ciclos de desnaturalización (a $94{ }^{\circ} \mathrm{C}$ durante 30 segundos), hibridación (a $61^{\circ} \mathrm{C}$ durante un minuto) y extensión (a $72{ }^{\circ} \mathrm{C}$ durante tres minutos), con una extensión final a $72{ }^{\circ} \mathrm{C}$ durante ocho minutos. La amplificación se verificó mediante electroforesis en gel de agarosa al $2 \%$ teñido con bromuro de etidio a 3,5 V/cm durante 30 minutos.

\section{Restricciones enzimáticas y análisis de la PCR- hsp70/RFLP}

Los productos obtenidos en todas las reacciones de PCR- $h s p 70$ se precipitaron en $70 \%$ de etanol absoluto y se suspendieron de nuevo en $10 \mu \mathrm{l}$ de agua bidestilada estéril. La restricción de los productos amplificados se hizo en un volumen total de $10 \mu \mathrm{l} \mathrm{a} 37^{\circ} \mathrm{C}$ durante toda la noche. Se utilizaron $5 \mu \mathrm{l}$ del ADN precipitado, $1 \mathrm{X}$ de la solución tampón

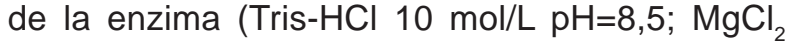
$10 \mathrm{~mol} / ; \mathrm{KCl} 100 \mathrm{~mol} / \mathrm{L}$; BSA 0,1 mg/ml), y $2 \mathrm{U}$ de la enzima Haelll (MBI Fermentas, St. Leon-Rot, Alemania), según las instrucciones del fabricante. Con base en los resultados, se hizo una segunda digestión enzimática siguiendo el algoritmo propuesto por Montalvo, et al. (15), utilizando $2 \mathrm{U}$ de las enzimas $\mathrm{Bccl}$ (New England Biolabs, Ipswich, M A, EUA) para discriminar L. panamensis de L. guyanensis, y Rsal (MBI, Fermentas) para diferenciar L. braziliensis de L. peruviana. La restricción con $R s a l$ permitió distinguir alelos diferentes de $L$. braziliensis en concordancia con lo reportado por Fraga, et al. (21). Para una mayor comprensión, la figura 1 muestra el flujograma de restricción seguido en este trabajo.

El tamaño de los fragmentos producidos por la restricción enzimática de los productos amplificados (PCR- $h s p 70 / R F L P)$, se determinó por electroforesis en gel de agarosa de alta resolución al $3 \%$ (Gentaur, Bruselas, Bélgica), a 3,5 V/cm durante tres horas. Se comparó la talla de las bandas obtenidas con las del marcador de peso molecular (Gene Ruler ${ }^{\text {TM }}$ 100bp DNA Ladder; $\mathrm{MBI}$, Fermentas, St Leon-Rot, Alemania). Se determinaron las bandas obtenidas de acuerdo con la enzima utilizada en las restricciones enzimáticas, tomando en cuenta los patrones característicos reportados previamente $(15,21)$. 


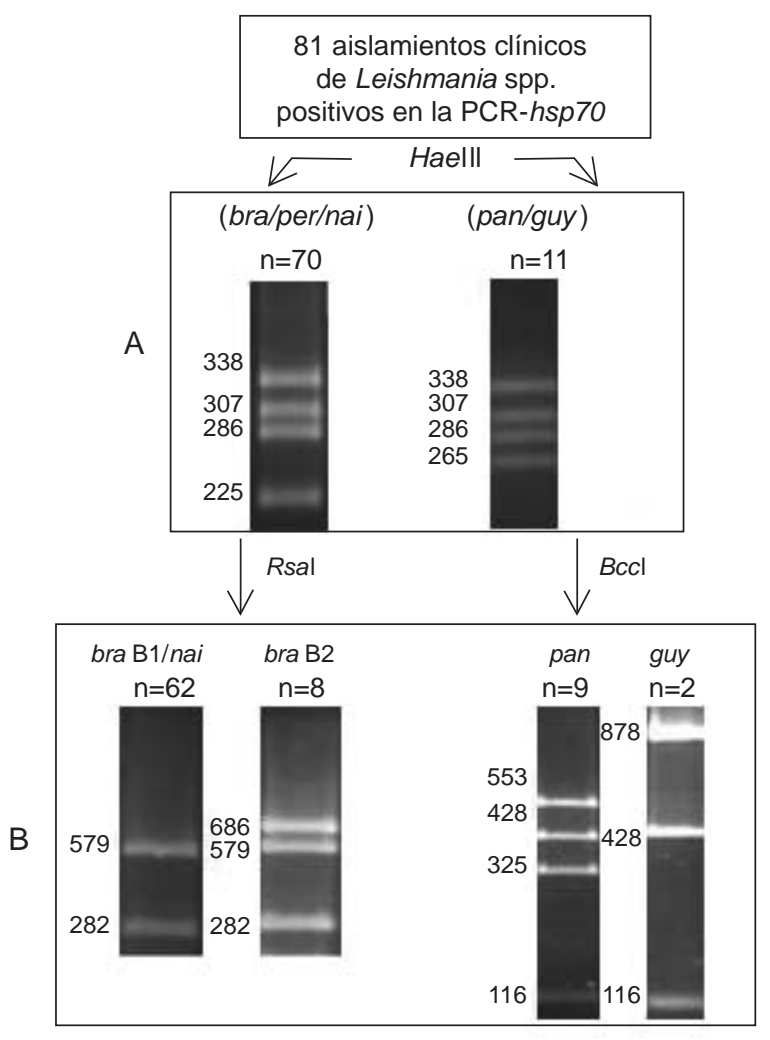

Figura 1. Patrones obtenidos tras las restricciones enzimáticas. A. La primera digestión (HaellI) produjo patrones comunes para L. braziliensis (bra), L. peruviana (per), L. naiffi (nai), así como para L. panamensis (pan) y L. guyanensis (guy). B. La segunda digestión (Rsal) produjo un patrón común para $L$. naiffi (nai) y el alelo B1 de L. braziliensis (bra B1), y otro para el alelo B2 de L. braziliensis (bra B2). La digestión con Bccl permitió la distinción entre $L$. panamensis y $L$. guyanensis con patrones individuales. Se indica el número de aislamientos identificados por cada especie (n) y el tamaño de los fragmentos obtenidos en cada figura. La denominación de alelos B1 y B2 concuerda con la propuesta de Fraga, et al. (21).

\section{Aspectos éticos}

En el momento de la toma de la muestra clínica se obtuvo el consentimiento informado de todos los pacientes para permitir que sus aislamientos clínicos se emplearan en el estudio y en otros proyectos de investigación.

\section{Resultados}

El ADN obtenido de los aislamientos resultó adecuado para hacer las amplificaciones mediante PCR-hsp70. Se obtuvieron los amplicones del tamaño esperado $(1,4 \mathrm{kpb})$ para todos los aislamientos clínicos de Leishmania spp. analizados provenientes de pacientes con leishmaniasis cutánea o mucocutánea. La precipitación posterior de los ácidos nucleicos permitió la restricción enzimática en todos los casos.
La digestión con Haelll produce patrones de bandas que pueden ser individuales, o compartidos por dos o más especies (20) y, en este caso, se obtuvieron los patrones correspondientes a dos grupos: $L$. braziliensis- $L$. peruviana- $L$. naiffi en 70 aislamientos y L. panamensis-L. guyanensis en los 11 restantes (figura 1). No se evidenciaron patrones relacionados con otras especies del género Leishmania.

Como no fue posible diferenciar especies individuales tras la primera digestión de los productos (figura 1A), se hizo una segunda restricción enzimática. La digestión con Rsal mostró que los 70 aislamientos digeridos presentaban un patrón característico de $L$. braziliensis- $L$. naiffi (con bandas de 579 pb y 282 pb) (figura 1B). En ocho de las 70 muestras se evidenció, además, una banda adicional de $686 \mathrm{pb}$ correspondiente a un patrón característico de un alelo diferente de $L$. braziliensis (figura 1B). En las 11 muestras restantes, la digestión con $\mathrm{Bccl}$ permitió identificar nueve aislamientos como $L$. panamensis y dos como $L$. guyanensis (figura 1B). El origen geográfico de los aislamientos y el número de especies identificadas se muestran en la figura 2 y en el cuadro 1.

\section{Discusión}

Las diferentes especies de Leishmania surgidas a lo largo de la evolución, e incluso algunas de sus variantes genéticas, se pueden identificar con el uso de herramientas moleculares como la PCR-RFLP. Esto representa una ventaja para las áreas endémicas donde existen varias especies, así como para el estudio de los casos importados $y$ de los que se presentan en los centros de referencia (22).

En años recientes el gen que codifica la proteína de choque térmico de $70 \mathrm{kDa}$ se ha ubicado entre las dianas más utilizadas para la detección y la tipificación de Leishmania spp. Con el protocolo diseñado por García, et al. (11), se han reportado nuevas restricciones enzimáticas basadas en la misma PCR, que permitieron distinciones aún no registradas de especies o grupos de especies. Así, se propusieron nuevas enzimas para distinguir entre las especies predominantes en Brasil, fundamentalmente del subgénero L. (Viannia) (23), y otras para identificar especies no estudiadas con anterioridad procedentes del Nuevo o del Viejo Mundo (15). Esta es la propuesta utilizada en este trabajo, la cual se aplica por primera vez al estudio de aislamientos clínicos de Leishmania spp. procedentes de distintas zonas de Colombia. 


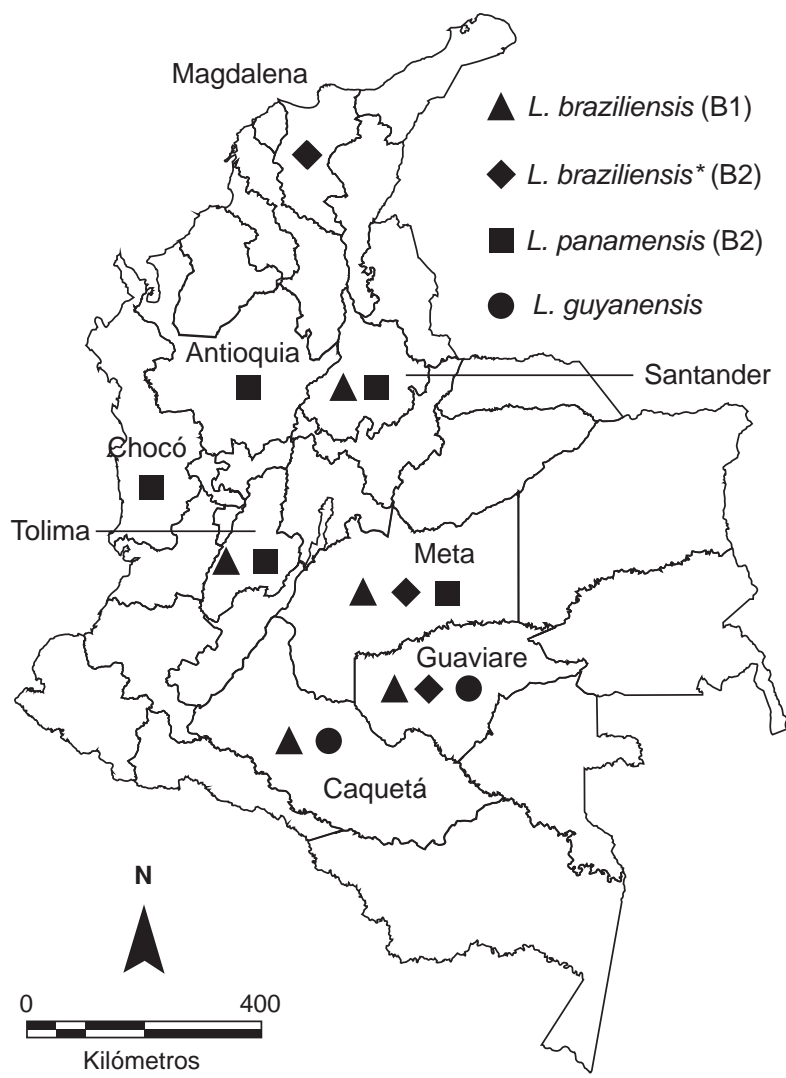

Figura 2. Ditribución geográfica de los aislamientos clínicos identificados mediante PCR-hsp70/RFLP

En relación con la identificación, la mayoría de los aislamientos correspondieron a L. braziliensis, la cual puede producir lesiones cutáneas o evolucionar hacia la aparición de síntomas nasales crónicos que resultan en la destrucción progresiva de las cavidades bucal y nasofaríngea, causando lesiones deformantes y dolorosas en una condición que se conoce como leishmaniasis mucocutánea (24). Incluso, se confirmó la enfermedad en un paciente tras 40 años de haber abandonado el área endémica (25), lo cual evidencia su importancia.
El $86,4 \%$ de los aislamientos se puede considerar como $L$. braziliensis con base en la presencia de diferentes alelos del gen hsp70 denominados L. bra (B1) y L. bra* (B2), de acuerdo con lo descrito por Fraga, et al. (21), al emplear el mismo procedimiento de PCR-RFLP en el análisis de muestras procedentes de Perú y Bolivia. En correspondencia con lo informado por estos autores, todos los aislamientos caracterizados en este estudio como $L$. braziliensis presentaron la banda característica de $579 \mathrm{pb}$, y unos pocos exhibieron, además, la banda adicional de 686 $\mathrm{pb}$ que cuando se presenta de forma individual identifica a $L$. peruviana $(15,21)$.

En este sentido, en un estudio de la evolución del complejo $L$. braziliensis mediante el análisis de la longitud de fragmentos polimórficos amplificados (Amplified Fragment Length Polymorphism, AFLP), en el cual se caracterizaron cepas de referencia y muestras clínicas, se demostró que, en la mayoría de los casos, L. braziliensis dispone de más de un alelo para el gen $h s p 70$ y que, solo en pocas ocasiones, muestra polimorfismo en el sitio Rsal utilizado en las digestiones propuestas. Por lo tanto, la mayoría de los aislamientos identificados tiene el genotipo de L. braziliensis y, otros, un genotipo que combina alelos de $L$. braziliensis y L. peruviana (26), pero no hay elementos que permitan considerarlos parásitos híbridos.

El origen geográfico de todos los aislamientos caracterizados como L. braziliensis concuerda con lo reportado por Corredor, et al. (17), a excepción de uno procedente de Tolima, donde los autores no reportaron su presencia.

Por otra parte, L. naiffi no se ha reportado en Colombia hasta el momento. Con anterioridad se había encontrado el polimorfismo intraespecie del mismo fragmento de hsp70 en tres secuencias nucleotídicas de $L$. naiffi, lo que permitió distinguirla de $L$. braziliensis mediante restricción enzimática

Cuadro 1. Origen geográfico y número de aislamientos de Leishmania identificados mediante PCR-hsp70/RFLP

\begin{tabular}{|c|c|c|c|}
\hline Especie identificada & Símbolo * & Procedencia geográfica de los aislamientos & Total \\
\hline L. braziliensis alelo B1 & & Meta (41), Caquetá (7), Guaviare (3), Santander (1), Tolima (1), ND (9) & 62 \\
\hline L. braziliensis alelo B2 & & Meta (4), Guaviare (1), Magdalena (1), ND (2) & 8 \\
\hline L. panamensis & & $\begin{array}{l}\text { Meta (1), Tolima (2), Antioquia (1), Santander (1), Chocó (1), Antioquia (1), } \\
\text { Antioquia (1), ND (1) }\end{array}$ & 9 \\
\hline L. guyanensis & & Guaviare (1), Caquetá (1) & 2 \\
\hline
\end{tabular}

* Ubicación geográfica en el mapa de la figura 2 ND: datos no disponibles 
(23). Más recientemente se han reportado algoritmos que permiten esta discriminación mediante la amplificación y la posterior digestión de fragmentos más cortos del mismo gen $h s p 70$, lo que se validó utilizando ADN de cuatro cepas de referencia (27).

Tomando en cuenta la notificación de los primeros casos de infección humana por $L$. naiffi en campos militares de entrenamiento en Ecuador, así como la presencia de esta especie en los posibles vectores asociados con la transmisión (28), sería recomendable evaluar los protocolos antes mencionados en un número mayor de cepas o diseñar identificaciones basadas en estudios de secuencias para determinar la presencia de $L$. naiffi y, en consecuencia, su papel clínico y epidemiológico en áreas donde hasta ahora no se conoce su circulación.

La discriminación entre $L$. panamensis y $L$. guyanensis usando la restricción del producto amplificado de la $h s p 70$ con $B c c l$, resulta muy evidente si se sigue el protocolo propuesto, el cual se evaluó previamente en cepas de referencia, aislamientos y muestras clínicas procedentes también de Colombia (16).

Leishmania panamensis se considera el principal agente etiológico de la leishmaniasis cutánea en Colombia y, también, se ha relacionado con casos puntuales de leishmaniasis cutánea diseminada (29), forma clínica atípica de poca distribución mundial y de mayor prevalencia en la zona norte de Brasil y la Amazonia colombo-brasilera. Esta especie, identificada en $11 \%$ de los aislamientos estudiados, es la más ampliamente distribuida en el país según Corredor, et al. (17). Otros autores identificaron esta especie mediante la metodología de anticuerpos monoclonales en $74 \%$ de las muestras procedentes de diversas regiones del país (30), incluidos los cuatro departamentos de donde provenían las muestras aquí identificadas.

Por otra parte, L. guyanensis se identificó solo en $2,4 \%$ de los aislamientos, todos procedentes del sudeste del país, lo que concuerda con otros estudios que reportaron esta especie en unas pocas muestras procedentes de los mismos departamentos de Guaviare $(17,30)$ y Caquetá $(17)$. Sin embargo, debe tenerse en cuenta la identificación previa de $L$. guyanensis como agente causal de leishmaniasis cutánea en Sucre, lo que constituyó el primer reporte de su presencia en la Costa Caribe colombiana (18). Este hallazgo también respalda el planteamiento de un posible cambio en el patrón de transmisión de esta especie en Colombia, la cual supondría su adaptación a condiciones ecológicas diferentes a las de las zonas selváticas donde habitualmente se encuentra, y su diseminación en áreas peridomiciliares, lo que es relevante para la identificación de casos y el tratamiento (31).

Otras variantes de PCR-RFLP basadas en la amplificación de distintas regiones del gen hsp70 $y$ evaluadas en diferentes contextos, resultan más sensibles y específicas para la detección e identificación de Leishmania spp. en muestras clínicas en zonas endémicas, y constituyen alternativas para la identificación individual de especies según el fragmento de gen que amplifican (32).

Por el contrario, la tipificación de aislamientos clínicos, o de otro tipo, en los que se puede obtener gran cantidad de ADN procedente del cultivo de los parásitos, no precisa de protocolos con gran sensibilidad. En ese caso, la amplificación del fragmento de $1.422 \mathrm{pb}$ del gen hsp70 utilizada en este estudio podría ser una alternativa atractiva, debido al número de especies individuales que permite identificar, y contribuir a los estudios epidemiológicos en el territorio colombiano u otras zonas endémicas que involucren cepas no caracterizadas provenientes de casos humanos, de vectores y de los posibles reservorios, para así tener una aproximación global al estudio de la leishmaniasis.

\section{Agradecimientos}

Los autores agradecen la colaboración prestada por Iván Darío Vélez y Jean Claude Dujardin en el desarrollo de este trabajo.

\section{Conflicto de intereses}

Los autores declaran que no existen conflictos de intereses en relación con este trabajo.

\section{Financiación}

Este trabajo fue financiado parcialmente por fondos recibidos del Programa DGOS de Cooperación para el Desarrollo a través de un proyecto de investigación conjunta del Instituto de Medicina Tropical de Amberes, Bélgica, y el Instituto de Medicina Tropical Pedro Kourí, La Habana, Cuba.

\section{Referencias}

1. Alvar J, Vélez ID, Bern C, Herrero M, Desjeux P, Cano $\mathbf{J}$, et al. Leishmaniasis worldwide and global estimates of its incidence. PLoS One. 2012;7:e35671. http://dx.doi. org/10.1371/journal.pone.0035671

2. Pérez-Ayala A, Norman F, Pérez-Molina JA, Herrero JM, Monge B, López-Vélez R. Imported leishmaniasis: 
A heterogeneous group of diseases. J Travel Medicine. 2009;16:395-401. http://dx.doi.org/10.1111/j.1708-8305.2009. 00341.x

3. Handman E, Elso C, Foote S. Genes and susceptibility to leishmaniasis. Adv Parasitol. 2005;59:1-75. http://dx.doi. org/10.1016/S0065-308X(05)59001-8

4. Schriefer A,Wilson ME, Carvalho EM. Recent developments leading toward a paradigm switch in the diagnostic and therapeutic approach to human leishmaniasis. Curr Opin Infect Dis. 2008;2:483-8. http://dx.doi.org/10.1097/ QCO.0b013e32830d0ee8

5. Rivas L, Moreno J, Canavate C, Alvar J. Virulence and disease in leishmaniasis: What is relevant for the patient? Trends Parasitol. 2004; 20:297-301. http://dx.doi. org/10.1016/j.pt.2004.05.005

6. Reithinger R, Dujardin JC. Molecular diagnosis of leishmaniasis: Current status and future applications. J Clin Microbiol. 2007;45:21-5. http://dx.doi.org/10.1128/JCM. 02029-06

7. Schönian G, Kuhls K, Mauricio IL. Molecular approaches for a better understanding of the epidemiology and population genetics of Leishmania. Parasitology. 2011;138:405-23. http://dx.doi.org/10.1017/S0031182010001538

8. Cupolillo E, Grimaldi G, Momen H, Beverley SM. Intergenic region typing (IRT): A rapid molecular approach to the characterization and evolution of Leishmania. Mol Biochem Parasitol. 1995;73:145-55.

9. Volpini AC, Passos VMA, Oliveira GC, Romanha AJ. PCR-RFLP to identify Leishmania (Viannia) braziliensis and L. (Leishmania) amazonensis causing American cutaneous leishmaniasis. Acta Trop. 2004;90:31-7. http://dx.doi.org/10. 1016/j.actatropica.2003.10.008

10. Ovalle-Bracho C, Porras de Quintana L, Muvdi Arenas S, Ríos-Parra M. Polymerase chain reaction with two molecular targets in mucosal leishmaniasis diagnosis: A validation study. Mem Inst Oswaldo Cruz. 2007;102:549-54. http://dx.doi.org/10.1590/S0074-02762007005000061

11. García L, Kindt A, Bermúdez H, Llanos-Cuentas A, De Doncker S, Arévalo J, et al. Culture-independent species typing of neotropical Leishmania for clinical validation of a PCR-based assay targeting heat shock protein 70 genes. J Clin Microbiol. 2004;42:2294-7. http://dx.doi.org/10.1128/ JCM.42.5.2294-2297.2004

12. Neves-Rocha M, Margonari C, Presot IM, Soares RP. Evaluation of 4 polymerase chain reaction protocols for cultured Leishmania spp. typing. Diagn Microbiol Inf Dis. 2010;68:401-9. http://dx.doi.org/10.1016/j.diagmicrobio. 2010.08.007

13. Monroy-Ostria M, Nasereddin A, Monteon VM, GuzmánBracho C, Jaffe CL. ITS1 PCR-RFLP diagnosis and characterization of Leishmania in clinical samples and strains from cases of human cutaneous leishmaniasis in states of the Mexican Southeast. Interdiscip Persp Inf Dis. 2014;2014:607287. http://dx.doi.org/10.1155/2014/607287

14. Buitrago R, Cupolillo E, Bastrenta B, Le Pont F, Martínez $\mathrm{E}$, Barnabé C, et al. PCR-RFLP of ribosomal internal transcribed spacers highlights inter and intra-species variation among Leishmania strains native to La Paz, Bolivia. Inf Gen Evol. 2011;11:557-63. http://dx.doi.org/10.1016/j. meegid.2010.11.019
15. Montalvo AM, Fraga J, Monzote L, Montano I, De Doncker S, Dujardin JC, et al. Heat-shock protein 70 PCR-RFLP: A universal simple tool for Leishmania species discrimination in the New and Old World. Parasitology. 2010;137:1159-68. http://dx.doi.org/10.1017/S0031182010000089

16. Montalvo AM, Fraga J, Montano I, Monzote L, Marín M, van der Auwera G, et al. Differentiation of Leishmania (Viannia) panamensis and Leishmania (V.) guyanensis using Bccl for hsp70 PCR-RFLP. Trans R Soc Trop Med Hyg. 2010; 104:364-7. http://dx.doi.org/10.1016/j.trstmh.2009.12.002

17. Corredor A, Kreutzer RD, Tesh RB, Boshell J, Palau MT, Cáceres E, et al. Distribution and ecology of leishmaniasis in Colombia. Am J Trop Med Hyg. 1990;42:206-14.

18. Martínez LP, Rebollo JA, Luna AL, Cochero S, Bejarano EE. Molecular identification of the parasites causing cutaneous leishmaniasis on the Caribbean coast of Colombia. Parasitol Res. 2010;106:647-52. http://dx.doi. org/10.1007/s00436-009-1712-6

19. Urbano J, Sánchez-Moreno M, Ovalle CE, Rosales MJ, Camargo YC, Gutiérrez-Sánchez R, et al. Characterization of cutaneous isolates of Leishmania in Colombia by isoenzyme typing and $k \mathrm{DNA}$ restriction analysis. Revista Ibero-Latinoamericana de Parasitología. 2011;70:16-24.

20. Sambrook J, Fritsch EF, Maniatis T. Molecular cloning. A laboratory manual. $2^{\text {nd }}$ edition. New York: Cold Spring Harbor Laboratory Press; 1989.

21. Fraga J, Veland N, Montalvo AM, Praet N, Boggild A, Valencia B, et al. Accurate and rapid species typing from cutaneous and mucocutaneous leishmaniasis lesions of the New World. Diagn Microbiol Inf Dis. 2012;74:142-50. http://dx.doi.org/10.1016/j.diagmicrobio.2012.06.010

22. Bañuls AL, Hide M, Prugnolle F. Leishmania and the leishmaniases: A parasite genetic update and advances in taxonomy, epidemiology and pathogenicity in humans. Adv Parasitol. 2007;64:1-109. http://dx.doi.org/10.1016/S0065$308 \times(06) 64001-3$

23. Alves da Silva L, Dos Santos de Sousa C, Cardoso da Graça G, Porrozi L, Cupolillo E. Sequence analysis and PCR-RFLP profiling of the $h s p 70$ gene as a valuable tool for identifying Leishmania species associated with human leishmaniasis in Brazil. Inf Gen Evol. 2010;10:77-83. http:// dx.doi.org/ 10.1016/j.meegid.2009.11.001

24. Weigle KA, Dávalos M, Heredia P, Molineros R, Saravia NO, D'Alessandro A. Diagnosis of cutaneous and mucocutaneous leishmaniasis in Colombia: A comparison of seven methods. Am J Trop Med Hyg. 1987;36:489-96.

25. Ruiz FL, Baquero JE. Leishmaniasis de la mucosa nasal en paciente de 66 años, con 40 años de vivir fuera de área endémica. Reporte de un caso. Acta de Otorrinolaringología y Cirugía de Cabeza y Cuello. 2011;39:39-42.

26. Odiwuor S, Veland N, Maes I, Arévalo J, Dujardin JC, van der Auwera G. Evolution of the Leishmania braziliensis species complex from amplified fragment length polymorphisms, and clinical implications. Infect Genet Evol. 2012;12:1994-2002. http://dx.doi.org/10.1016/j. meegid.2012.03.028

27. Fraga J, Montalvo AM, Maes I, Dujardin JC, van der Auwera G. Hindll and Sdul digests of heat-shock protein 70 PCR for Leishmania typing. Diagn Microbiol Infect Dis. 2013;77:245-7. http://dx.doi.org/10.1016/j.diagmicrobio. 2013.07.023 
28. Kato H, Calvopiña M, Criollo H, Hashiguchi Y. First human cases of Leishmania (Viannia) naiffi infection in Ecuador and identification of its suspected vector species. Acta Trop. 2013;128:710-3. http://dx.doi.org/10.1016/j. actatropica.2013.09.001

29. Rincón MY, Silva SY, Dueñas RE, López-Jaramillo P. Leishmaniasis cutánea diseminada: reporte de dos casos en Santander, Colombia. Rev Salud Pública. 2009;11:14550. http://dx.doi.org/10.1590/S0124-00642009000100015

30. Ovalle CE, Porras L, Rey M, Ríos M, Camargo YC. Distribución geográfica de especies de Leishmania aisladas de pacientes consultantes al Instituto Nacional de
Dermatología Federico Lleras Acosta, E.S.E., 1995-2005. Biomédica. 2006;26:145-51. http://dx.doi.org/10.7705/ biomedica.v26i1.1508

31. Rodríguez-Barraquer I, Góngora R, Prager M, Pacheco R, Montero LM, Nevas A, et al. Etiologic agent of an epidemic of cutaneous leishmaniasis in Tolima, Colombia. Am J Trop Med Hyg. 2008;78:276-82.

32. Montalvo AM, Fraga J, Maes I, Dujardin JC, van der Auwera G. Three new sensitive and specific heat-shock protein 70 PCRs for global Leishmania species identification. Eur J Clin Microbiol Infect Dis. 2012;31:1453-61. http:// dx.doi.org/10.1007/s10096-011-1463-z 